\title{
Pressure Coupling Of the Spherical Linear Eddy Model to RANS-CFD for Internal-Combustion Engine Simulation.
}

\author{
Nidal Doubiani' ${ }^{1}$, Abhilash Menon ${ }^{1}$, Alan R. Kerstein ${ }^{2}$, Michael Oevermann ${ }^{3}$ \\ Chalmers University of Technology, \\ ${ }^{1}$ Dpt of Mechanics and Maritime Sciences, Div of Combustion and Propulsion systems \\ , Gothenburg, Sweden \\ nidal@chalmers.se; menona@chalmers.se \\ 272 Lomitas Road \\ Danville, CA, USA \\ alan.kerstein@gmail.com \\ ${ }^{3}$ Brandenburgische Technische Universität (BTU) Cottbus-Senftenberg, Germany and \\ Chalmers University of Technology, Gothenburg, Sweden \\ michael.oevermann@b-tu.de
}

\begin{abstract}
As a result of the increase of the use of combustion engines, more restrictive emission standards are applied. New combustion technologies are being constantly developed to enhance the internal combustion engine, this is motivated by overcoming issues that are relevant for both engine efficiency and the environmental aspects. To do that, turbulent combustion modelling is needed.

In this paper, an updated version of the Linear Eddy Model (LEM) will be presented. LEM is capable of simulating premixed, nonpremixed and mixed mode combustion, and it is a regime independent model that runs under the assumption of finite-rate chemistry that is sensitive to turbulent chemistry interactions, which makes it suitable for prediction of pollutant formation. A New coupling scheme to the CFD-RANS simulation is proposed, the coupling is based on linking the two models via pressure. The benefits of pressure coupling are that the effects of wall heat losses and latent heat of evaporation that are modelled on the CFD side are an intrinsic part of the pressure term that is linking the two models, in contrast to volume coupling in which these relevant phenomena need supplementary modelling on the LEM side. The pressure coupling results in a radially uniform dilatation of the LEM domain reflecting the combined effects of pressure change, fuel addition, and cylinder volume change during the engine cycle. Consistency of the LEM cone volume and the CFD domain volume is maintained by adopting a split operator strategy involving a volume correction that adjusts the cone angle. A Spherical Stand-Alone Linear Eddy Model (SSALEM) has been created to conduct relatively fast simplified code development and parameter studies. SSALEM input parameters were drawn from a WSR-RANS simulation and a ID slider-crank model that calculates the combustion chamber volume that corresponds to a given crank-angle. Model results show the capability to physically track the evolution of several scalars that are solved on the LEM line such as temperature, fuel, and intermediate species in the combustion process.
\end{abstract}

Keywords: Linear Eddy Model - Pressure coupling - Stand Alone Model - Volume adaption

\section{Introduction}

Considering the increase in pollutant emissions due to the surge of the use of internal combustion engines, new combustion techniques such as homogeneous charge compression ignition (HCCI), reactivity control combustion ignition (RCCI) and high levels of exhaust gas recirculation (EGR) require further development. Turbulent combustion modelling is considered as a crucial tool for the development of these combustion techniques.

Several combustion models have been used and developed during the last years for classical usage such as gasoline combustion (premixed) or diesel combustion (non-premixed). They are typically limited to these two combustions. For example, flamelet models [1] assume the formation of laminar flame structures in a turbulent flow field, resulting in limited interaction between chemistry, molecular transport, and turbulence in the flamelet models because of the parametric coupling that these models are based on. The coupling in flamelet models depends on scalar dissipation rate for non-premixed cases and mean turbulent velocities for the premixed cases.

The Linear Eddy Model has been used a sub-grid model to overcome this limitation. It is a regime and mode independent model that is based on the statistical representation of flow time advancement along a physical line of sight represented by a 
one-dimensional computational domain. Because LEM captures direct interactions between turbulent advection, diffusion, and chemical reactions, it can predict highly unsteady effects such as extinction and re-ignition without supplementary modelling. It can be used in different geometries (planar, cylindrical, or spherical) depending on the application case requirements. LEM can be coupled as a sub-grid model in several ways. It has been used in the context of LES simulations in the LES-LEM approach [2] which is a one-dimensional representation of the turbulent combustion process in every LES cell with spatial and temporal resolution comparable to direct numerical simulations. The disadvantage of the LES-LEM model is the computational cost of this high resolution.

In the context of this paper, one LEM line represents the entire combustion chamber, thereby greatly reducing the computational cost. A previous version of this formulation was termed RILEM [3], which stands for the representative interactive linear eddy model. In this model the coupling was done based on a volume constraint, which required supplementary modelling of the heat effects on the LEM side. However, in this paper, LEM will be presented in a oneway-coupled form in which the results of a WSR-RANS simulation supply the pressure time history to LEM but LEM does not provide chemical or other closure information to the CFD.

\section{Mathematical Model}

The Linear Eddy Model $[4,5]$ was proposed originally as a scalar mixing model for non-reactive flows. It was later extended to describe reactive flows. It consists of two main processes. The first process is the turbulent transport, represented by a stochastic sequence of independent eddy events. The second process time advances all other effects, which are diffusion, and chemical reactions in the present application. The Linear Eddy Model provides affordable full resolution of spatial and temporal scales by solving the reactive zero-Mach number equations on a one-dimensional line of sight.

\subsection{LEM Diffusion and Chemical reactions}

The LEM code is based on a pre-existing C++ implementation of One-Dimensional Turbulence (ODT) by David Lignell [4]. The ODT code offers certain features that are adopted in the LEM framework such as grid adaptation and a Lagrangian formulation of the balance equations for species mass fractions $Y_{S}$ and enthalpy $h$. The balance equations are the following:

$$
\begin{gathered}
\rho \frac{D Y_{s}}{D t}=-\frac{d j_{s}}{d x}+M_{s} \dot{w}_{s} \\
\rho \frac{D h}{D t}=-\frac{d p}{d t}-\frac{d q}{d x}-\sum_{s} j_{s} \frac{d h_{s}}{d x}-\sum_{s} h_{s} M_{s} \dot{w}
\end{gathered}
$$

$\rho$ is density, $j_{s}$ the species diffusion flux, $M_{s}$ the species molar mass, $p$ the pressure, $q$ the heat flux, $h_{s}$ the enthalpy of species s including the heat formation heat, and $\dot{w}_{s}$ the chemical source term. The temperature is calculated using the caloric equation of state.

$$
h(T)=\Delta h_{S}^{0}+\int_{T 0}^{T} c_{p, s}(T) d T
$$

where $\Delta h_{s}^{0}$ is the standard heat of formation of species s and $c_{p}$, denotes the mass specific heat capacity at constant pressure. The continuity equation in the lagrangian formulation for the one-dimensional line is $\rho \Delta x=$ const.

The above-mentioned balance equations are solved using a second- order accurate scheme with central discretization. 
of the diffusion terms. The chemistry solver that is used for chemical reactions is the CVODE package using the stiff chemical source integrator an implicit BDF Method. The chemistry is solved in parallel using open MP, where it is possible to specify the number of threads on which the chemistry will be solved.

\subsection{LEM Turbulent Transport}

The turbulence advection in LEM is represented by stochastic eddy events. An eddy event in the context of LEM is a so-called "Triplet Map." This operation is a rearrangement of the scalars that constitute the state of each LEM cell. It mimics the effect of turbulent vortices. In addition to the usual LEM representation of the turbulent cascade, triplet maps are also used to represent large-scale fluid motions inside the combustion chamber.

\subsubsection{Small Scale Turbulence}

The triplet map process follows the presented steps: Once the eddy is decided to be implemented on a certain region of the line, that section of the line will be copied three times, the middle copy gets inverted, the three copies are then connected back with each other and finally the result is compressed back to the original length of the eddy. The described operation increases the gradient within the interval where the eddy happened similarly to the effect generated by the compressive strain in a turbulent flow.

Three quantities are needed to specify an eddy event, size of the eddy $l$, the eddy's location and an eddy event time. In the LEM the eddy size is sampled from a prescribed size distribution. Assuming Kolmogorov inertial-range scaling [5], the eddy distribution is

$$
f(l)=\frac{5}{3} \frac{l^{-8 / 3}}{\eta^{-5 / 3}-l_{t}^{-5 / 3}}
$$

Using the turbulent Reynolds number

$$
R e_{t}=\frac{u^{\prime} l_{t}}{v}
$$

where $v$ is the kinematic viscosity and $u^{\prime}$ is the root mean square velocity fluctuation, $l_{t}$ the integral length scale, the Kolmogorov length scale $\eta$ is determined from the inertial scaling law.

$$
\eta=N_{n} L R e_{t}^{-\frac{3}{4}}
$$

The mean number of eddy events per unit time per unit length of the 1D domain is

$$
\lambda=\frac{54}{5} \frac{v R e}{C_{\lambda} l_{t}^{3}} \frac{\left(l_{t} / \eta\right)^{5 / 3}-1}{1-\left(\eta / l_{t}\right)^{4 / 3}}
$$

where $C_{\lambda}=15$ and $N_{\eta}=10.76$ are model constants with values that can be found in the literature [6]. The turbulent diffusivity on the LEM line corresponds to

$$
D_{t}=u^{\prime} l_{t} / C_{\lambda}
$$


Eddy locations, where location is nominally defined as the location of left boundary of an eddy, are randomly sampled from a uniform distribution of eddies over the line and the eddy time is sampled under the assumption of a Poisson process with a mean eddy occurrence time.

$$
\Delta \tau_{E d d y}=(\lambda L)^{-1}
$$

where $\mathrm{L}$ is the domain size. For boundary conditions other than periodic, sampled eddies that extend beyond the right boundary of the domain are not implemented.

\subsubsection{Large Scale Turbulence}

It is possible to implement large scale turbulence effects such as swirl and tumble on the LEM line, like what happens in the context of real engines. These effects are represented by big eddies that are introduced to the LEM line to obtain sufficient dispersion of fuel from the vapor deposition region to other regions of the domain. The big eddies respect a constant size which has been chosen to be equal to the half of the combustion chamber bore.

Like the small eddies, big eddies are randomly sampled uniformly along the LEM line. A big-eddy time scale is calculated based on the exit velocity of the fuel $v_{n}$ and engine cylinder length scale $l_{c}$ using

$$
\tau_{B i g E d d y}=\theta \frac{v_{N}}{l_{c}}
$$

where $\theta$ is a mixing time constant that accounts for the effect of different geometries and flow conditions on the mixing. Previously and in this study, $\theta$ is set equal to unity. The number of the large eddies that would happen in the entire simulation is fixed at the beginning to be the time for one engine cycle divided by the big-eddy time scale and their occurrence times are independently sampled based on a uniform distribution of occurrence time during one engine cycle.

This sampling process of the large eddies is different from one used for the small eddies to avoid excessive cycle to cycle variability of the fuel dispersion process that can be created from significant cycle variability of the number of large eddies.

\subsection{LEM Pressure Coupling}

LEM and CFD can be coupled in two different ways: i) with volume coupling that was applied in the previous version [3] and ii) via pressure coupling which has been developed and implemented in the current project so far.

In the pressure based CFD-LEM coupling the pressures on the CFD and the LEM match while the volume can (and will) slightly deviate. This is achieved by imposing the CFD pressure on the LEM. The main advantage of the pressure coupling compared to the volume-based coupling is that relevant phenomena such as latent heat of evaporation and wall heat losses, which are modelled in detailed 3D geometry on the CFD side, are implicitly encoded into the pressure. The pressure coupling therefore allows consistent LEM treatment of those effects, which otherwise would require additional modelling on the LEM side.

\subsection{Split Operator Strategy}

In the pressure-based coupling we enforce a spatially constant LEM pressure matching the spatially averaged time dependent pressure of the CFD. This can be achieved in several different ways, here we adopt the following strategy which we call the split operator strategy:

1. Implementation of physical processes (fuel injection, combustion, diffusion, etc.) as constant volume processes in each LEM cell. This will lead to a change of pressure in each individual LEM cell.

2. Isentropic compression of each LEM cell to the target CFD pressure under the assumption of a fixed composition. This step will alter pressure, temperature, and density within each LEM cell.

3. Calculation of the total volume of the LEM domain. 
4. Adjusting the cone-angle of the LEM domain with a prescribed length of the LEM domain to match the volume calculated in step 3 .

5. Re-distribution of cell boundaries on the LEM domain.

In the following we describe some of the steps mentioned above in more detail.

\subsubsection{LEM Volume Adjustment}

As mentioned above, the LEM domain is a double cone. Therefore, the volume of the LEM domain can be adjusted by changing the length of the cone and/or the cone angle. Here we adjust the volume by changing the cone angle and keeping the length fixed. This is motivated by the assumption that the cone is oriented in direction of a spray jet and therefore, under this assumption the length of one LEM cone is limited by the distance between the injector and the cylinder walls.

If $\mathrm{D}$ denotes a characteristic length of the cylinder, e.g., the bore or the stroke, the cone angle $\alpha$ is given by:

$$
\alpha=\operatorname{acos}\left(1-\frac{12 V}{\pi D^{3}}\right)
$$

where $V_{L E M}$ is the total volume of the LEM line, which is known after step 3 of the split-operator approach. Since we know the volume of each individual LEM cell, the position of the cell faces on the LEM domain can be re-calculated.

\subsubsection{Fuel deposition on the LEM line}

Fuel injection is modelled in detail on the CFD side via the spray model. However, evaporated fuel on the CFD domain needs to be added to the LEM line which only represents the gas phase. For this, two main parameters are extracted from the CFD side to be imposed on the LEM to represent the fuel injection process: i) the evaporated fuel mass in one CFD time step and ii) the total volume of cells where fuel has been evaporated. In principle it would be possible to implement a spray model on the LEM line itself, however, it is easier and probably more consistent to use information from the CFD spray model. To have a consistent representation of quantities from the CFD to the LEM, a volume ratio between the CFD and LEM is defined as

$$
Q=\frac{V_{L E M}}{V_{C F D}}
$$

which is used to scale the vapor deposition volume between the CFD and the LEM.

Evaporated fuel on the LEM line is injected symmetrically relative to the meeting point of the two cones. The current fuel deposition strategy matches the total volume of the cells with and without evaporation on the CFD and the LEM side. On the CFD we evaluate the volume ratio of cells with evaporation to the total volume as

$$
\beta=\frac{\sum V_{e v, i}}{V_{C F D}}
$$

This ratio defines an initial guess of the volume and the associated radius $R_{L E M}^{i n j}$ around the center of the LEM domain over which fuel on the LEM line is injected. The actual fuel mass deposited in each cell depends on its volume based on

$$
m_{e v, i}^{L E M}=m_{e v}^{C F D} \cdot Q \cdot \frac{V_{e v, i}^{L E M}}{\sum V_{e v, i}^{L E M}}
$$

The implemented fuel deposition method does not guarantee that the fuel vapor mass in each cell stays below the saturation level. However, so far this has not been a problem in the simulations, but it is straightforward to extend the fuel deposition strategy to take local saturation levels into account. 
After vapor deposition new values for density, mass-fractions, and temperature in each affected cell need to be updated applying a simple mixing process at constant volume (the volume of the LEM cell). For the new LEM cell density in cell i we have

$$
\rho_{\text {new }, i}=\rho_{\text {old }, i}+\rho_{\text {Fuel }, i} \cdot \frac{V_{\text {Fuel }, i}}{V_{L E M, i}}
$$

and for the updated mass-fractions

$$
Y_{s, \text { new }, i}=\frac{Y_{s, \text { old }, i} \cdot m_{\text {old }}+Y_{s, \text { add }, i} \cdot m_{\text {add }}}{m_{\text {old }}+m_{\text {add }}}
$$

The temperature of the mixture is calculated with a simplified mixing rule ignoring the difference in specific heats of the added fuel vapor and the mixture within the LEM cell:

$$
T_{\text {new }}=\frac{T_{\text {old }} \cdot m_{\text {old }}+T_{\text {Fuel }} \cdot m_{\text {Fuel }}}{m_{\text {old }}+m_{\text {Fuel }}}
$$

Due to the mixing process LEM cells with fuel injection have changed density, composition and temperature, therefore they have changed pressure as well. To have a constant pressure on the line we correct the pressure and adjust the LEM cell faces and the cone angle according to the split operator approach described above.

\section{Spherical Stand-Alone Linear-Eddy Model (SSALEM)}

A stand-alone engine LEM model has been created. The stand-alone model offers the following possibilities: simplified code development and validation, faster computation to enable many parameter variations, and modularity for easy coupling to any combustion CFD software.

The time-varying driving parameters for the LEM model (turbulence parameters, cylinder pressure traces and evaporated fuel mass deposition rate and penetration distance) can be provided by simple models or - as done here - with a separate CFD simulation based on the Well Stirred Reactor (WSR) in a pre-processing step. A standard 1D slider-crank model has been added to the stand alone LEM that calculates the volume of the combustion chamber as a function of the engine speed.

\section{Results}

\subsection{Case Description : Volvo Heavy Duty Engine}

The investigated case is a single cylinder of a Volvo 13L six-cylinder heavy-duty truck engine. It has a high-pressure fuel injector located in the centre of the top of the combustion chamber and a compression ratio of 15.8:1. The simulation was on a part load configuration with the following operating conditions initial pressure 1.69 bar, initial temperature $395 \mathrm{~K}$, IMEP 7.08 bar, engine speed $1200 \mathrm{RPM}$, start of injection $3.1^{\circ} \mathrm{bTDC}$, end of injection $3.3^{\circ}$ aTDC, injected mass $13 \mathrm{mg}$, and initial gas composition by mass $\mathrm{O} 216.5 \%, \mathrm{~N} 275.3 \%, \mathrm{CO} 25.97 \%, \mathrm{H} 2 \mathrm{O} 2.26 \%$. The chemical mechanism that was used for this study is a reduced mechanism for n-dodecane combustion which involves 54 species and 256 chemical reactions [7].

\subsection{Results}

The input parameters for running the SSALEM were created by running a Well Stirred Reactor (WSR) RANS simulation of the case, which is based on Lib-ICE, which is a set of OpenFOAM libraries and solvers for internal combustion engines which is implemented by the ICE group at Politecnico di Milano. Once the inputs are generated, the SSALEM is simulated.

As described before, the stand-alone spherical LEM has been created to investigate different parameters on the LEM line for faster simulations. The possibility to represent the LEM line in physical space has also been added to the code, which 
provided the ability to investigate in detail the different processes that modify the LEM physical state and geometrical structure. The aim of this simulation is to observe the output that SSALEM can provide.

To describe the evolution of the LEM line, the temperature and the $n$-dodecane mass fraction were chosen to represent the different states the LEM line is experiencing. The following two figures display the distribution of temperature, fuel mass fraction, and carbon monoxide mass fraction on the LEM line.

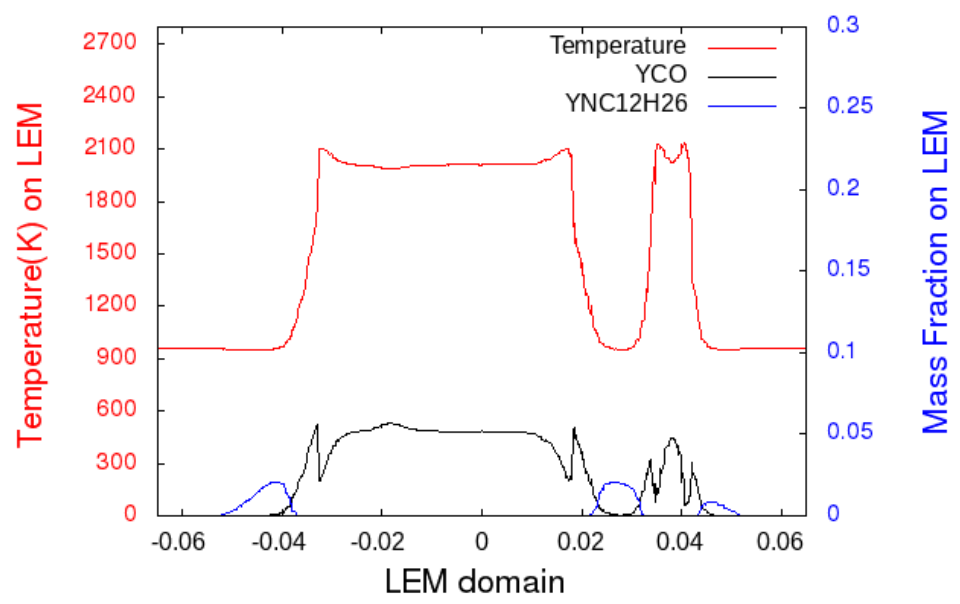

Fig 1: Snapshot of the LEM solution at CAD $=360.34$

In Fig 1, the simulation has reached the crank-angle 360.34, It is seen that the LEM line is relatively combusted with the highest temperature on the line reaching $T_{L E M_{M a x}}=2100 \mathrm{~K}$. It also appears that the fuel is decreasing which corresponds to the consumption of the fuel due to combustion.

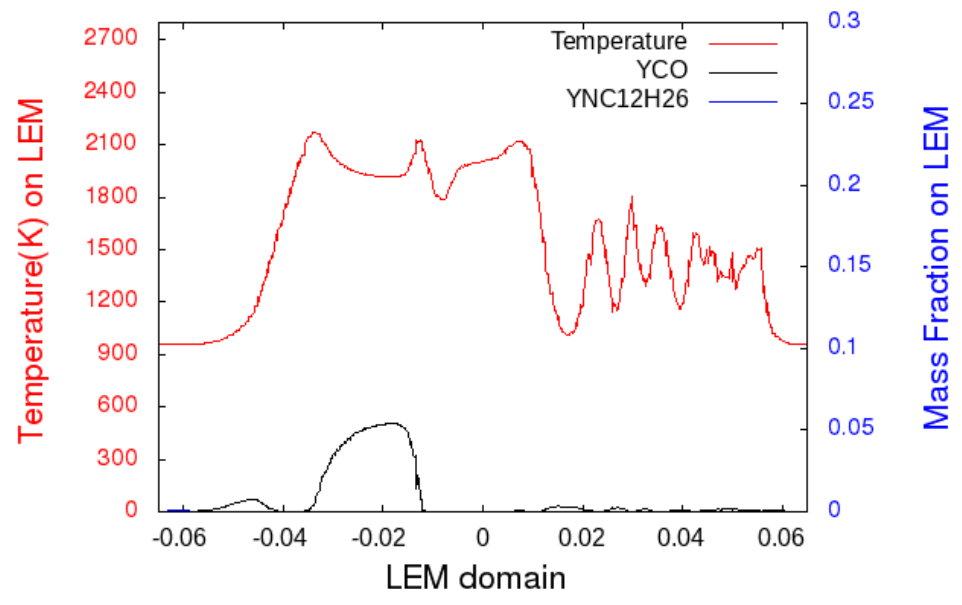

Fig 2: Physical representation of a developed LEM line CAD $=363.85$

The combination of the small-scale turbulence, the large-scale turbulence, the diffusion, and the output of the chemistry solver resulted in the state of the LEM line represented above in Fig 2. The LEM representation tool provided the possibility to visually verify the state of the line. The split operator strategy appears to be working by maintaining the LEM length be equal to the combustion chamber bore diameter. 


\section{Conclusion}

Based on the reported results, the pressure-coupled SSALEM can potentially be used as a combustion model by linking it to a CFD-RANS simulation, which is a promising strategy to explore. LEM's sensitivity to turbulence chemistry interactions potentially enables investigation and prediction of pollutant formation.

The development of the SSALEM is an important step in the construction of the pressure-coupled RANS-LEM thanks to its efficiency in code debugging and its clarity in displaying the important steps of the model's framework. It is also useful as a physical representation of the regime evolution along the one-dimensional line during the engine cycle, which can be used for parameter investigation.

LEM is to be coupled with CFD using a pressure constraint. The scalars that are solved on the SSALEM level will be conditionally based on a CFD-level model of the joint PDF of mixture fraction and progress variable. The conditioning will generate outputs that will be mapped to each CFD computational cell. Coupling the LEM with the CFD will result in a different link between the two solvers in terms of pressure than in SSALEM. In the current configuration pressure is precalculated using conventional CFD, while in the coupled scheme the pressure will be time advanced at the CFD level after each time interval of LEM advancement, resulting in interesting phenomena that will appear on both the CFD and the LEM sides. These phenomena are to be investigated in future work.

\section{Acknowledgements}

N. D and M. O thank Chalmers Combustion Research Center (CERC) for their financial support. The authors thank Andreas Nygren for his contribution to this work.

\section{References}

[1] H. Pitsch, M. Chen, and N. Peters, "Unsteady flamelet modeling of turbulent hydrogen-air diffusion flames," Symposium (International) on Combustion/The Combustion Institute, vol. 27, pp. 1057-1064, 1998.

[2] S. Arshad, B. Kong, A. R. Kerstein, and M. Oevermann, "A strategy for large-scale scalar advection in large eddy simulations that use the linear eddy sub-grid mixing model," International Journal of Numerical Methods for Heat \& Fluid Flow, vol. 10, pp. 2463-2479, 2018.

[3] T. Lackmann, A. R. Kerstein, and M. Oevermann, "A representative linear eddy model for simulating spray combustion in engines (RILEM)," Combustion and Flame, vol. 193, pp. 1-15, 2018.

[4] D. O. Lignell, A. R. Kerstein, G. Sun, and E. I. Monson, "Mesh adaption for efficient multiscale implementation of onedimensional turbulence," Theoretical and Computational Fluid Dynamics, vol. 27, pp. 273-295, 2013.

[5] A. R. Kerstein, "Linear-eddy modeling of turbulent transport. II: Application to shear layer mixing," Combustion and Flame, vol. 75, pp. 397-413, 1989.

[6] T. M. Smith and S. Menon, "One-dimensional simulations of freely propagating turbulent premixed flames," Combustion Science and Technology, vol. 128, pp. 99-130, 1997.

[7] T. Yao, Y. Pei, B.J. Zhong, S. Som, and T. Lu, "A compact skeletal mechanism for n-dodecane with optimized semiglobal low-temperature chemistry for diesel engine simulations," in 9th U.S. National Combustion Meeting, 2015. 